Supporting Information

\title{
Impacts of solvent and alkyl chain length on the lifetime of singlet cyclopentane-1,3-diyl diradicaloids with $\pi$-single bonding
}

\author{
Qian Liu, Zhe Wang, and Manabu Abe* \\ Department of Chemistry, Graduate School of Science, Hiroshima University, 1-3-1 Kagamiyama, Higashi- \\ Hiroshima, Hiroshima 739-8526, Japan. \\ E-mail: mabe@hiroshima-u.ac.jp
}

\section{Table of Contents}

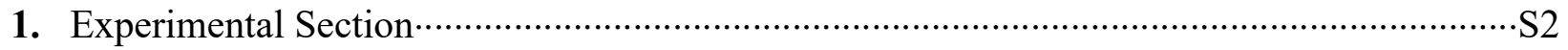

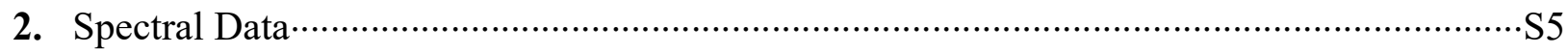

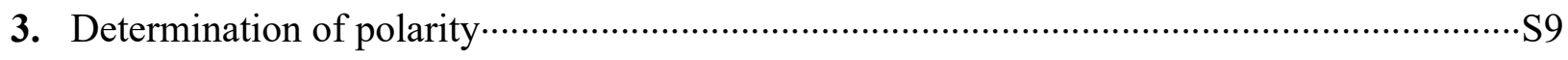

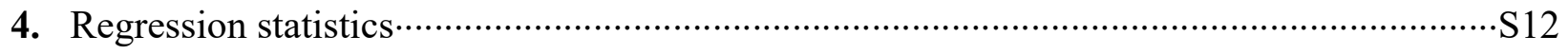

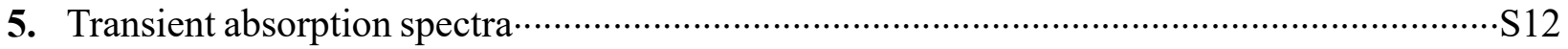




\section{Experimental Section}

All commercially available reagents were purchased from TCI, Wako, Sigma Aldrich and Kanto and were used without further purification. NMR spectra were recorded on a Bruker Ascend $400\left({ }^{1} \mathrm{H}\right.$ NMR: 400 $\mathrm{MHz},{ }^{13} \mathrm{C}$ NMR: $100 \mathrm{MHz}$ ) spectrometer at $298 \mathrm{~K}$ and referenced to the residual solvent peak. Coupling constants $(J)$ are denoted in $\mathrm{Hz}$ and chemical shifts $(\delta)$ in ppm. The abbreviations $\mathrm{s}, \mathrm{d}, \mathrm{t}$, and $\mathrm{m}$ stand for the resonance multiplicities singlet, doublet, triplet and multiplet, respectively. Mass spectrometric data were measured with Thermo Fisher Scientific LTQ Orbitrap XL and JMS-T100GCV AccuTOF GCv. UV-vis spectra were recorded on a SHIMADZU UV-3600 Plus spectrometer with a slit width of 1 and $0.01 \mathrm{~nm}$. The excitation source for sub-microsecond laser flash photolysis was a tunable Nd:YAG minilite laser at $355 \mathrm{~nm}$. The monitoring system consisted of a $150 \mathrm{~W}$ xenon arc lamp as light source, a Unisoku MD200 monochromator detection and a photomultiplier. The temperature was controlled by Unisoku CoolSpek USP-203-B. The excitation source was a passive Q-SWITCH microchip laser at $355 \mathrm{~nm}$.

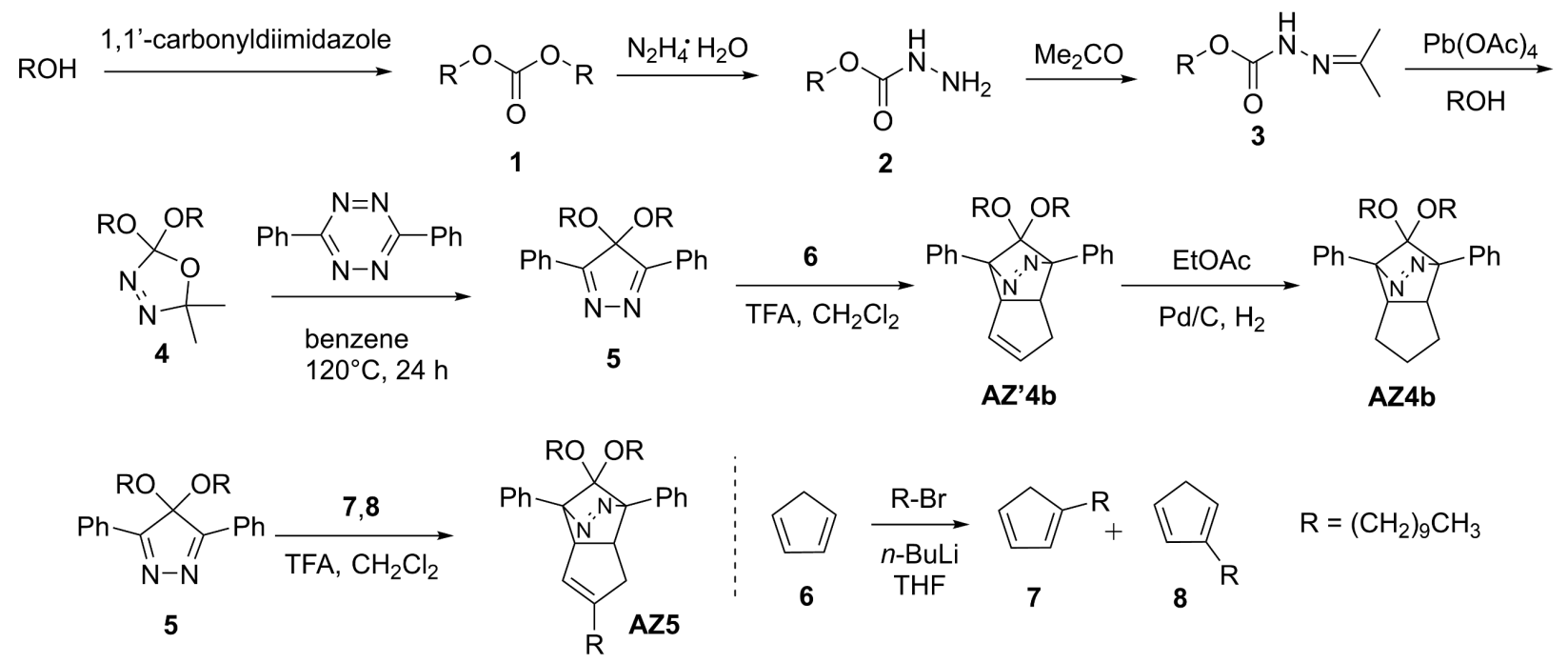

Scheme S1. Synthesis of azoalkanes AZ4b and AZ5.

Didecyl carbonate 1

1,1'-carbonyldiimidazole (4.00 g, $24.68 \mathrm{mmol}, 1$ equiv) was added followed by 1-decanol (19 mL, 4 equiv) at room temperature under nitrogen atmosphere. After stirring for $22 \mathrm{~h}$ at $55^{\circ} \mathrm{C}$ (oil bath), the reaction was 
quenched with saturated brine and extracted with EtOAc. The combined organic layers were dried over $\mathrm{Na}_{2} \mathrm{SO}_{4}$, filtered and concentrated resulting in crude which was used directly for the preparation of decyl hydrazinocarboxylate 2 .

Decyl hydrazinocarboxylate 2

Crude didecyl carbonate 1 including 1-decanol and hydrazine hydrate (1.64 mL, 1.5 equiv) was stirred for 1 $\mathrm{h}$ at room temperature under nitrogen atmosphere and then heated to $80^{\circ} \mathrm{C}$ (oil bath). After $20 \mathrm{~h}$, the reaction mixture was cooled to room temperature and washed by aq $\mathrm{NaHCO}_{3}$ and $\mathrm{H}_{2} \mathrm{O}$ and extracted with EtOAc. Drying $\left(\mathrm{Na}_{2} \mathrm{SO}_{4}\right)$, filtration, evaporation of solvent and purification by column chromatography on silica gel (hexane : EtOAc = 1: 1) afforded decyl hydrazinocarboxylate 2 ( $4.20 \mathrm{~g}, 19.41 \mathrm{mmol}, 78.7 \%$ of 2 steps) as white solid. ${ }^{1} \mathbf{H}$ NMR (400 MHz, $\left.\mathrm{CDCl}_{3}\right): \delta=5.88(\mathrm{~s}, 1 \mathrm{H}), 4.10(\mathrm{t}, J=6.75 \mathrm{~Hz}, 2 \mathrm{H}), 3.73(\mathrm{~d}, J=3.35 \mathrm{~Hz}$, 2H), $1.65-1.58(\mathrm{~m}, 2 \mathrm{H}), 1.36-1.21(\mathrm{~m}, 14 \mathrm{H}), 0.88(\mathrm{t}, J=7.00 \mathrm{~Hz}, 3 \mathrm{H})$

3

A mixture of decyl hydrazinocarboxylate $2(0.82 \mathrm{~g}, 3.78 \mathrm{mmol})$ and $\mathrm{Na}_{2} \mathrm{SO}_{4}(0.81 \mathrm{~g}, 1.5$ equiv) was stirred at room temperature under nitrogen atmosphere in acetone $(7 \mathrm{~mL})$ for overnight. The reaction mixture was filter and concentrated to give compound $\mathbf{3}$ as orange solid $(0.96 \mathrm{~g}, 3.74 \mathrm{mmol})$, which was directly used for next step.

4

A two-neck flask was charged with lead tetraacetate ( $2.00 \mathrm{~g}, 1.2$ equiv), 1-decanol (2.2 mL, 3.1 equiv) and $\mathrm{CH}_{2} \mathrm{Cl}_{2}(1.6 \mathrm{~mL})$ under nitrogen atmosphere. Then $3(0.96 \mathrm{~g}, 3.74 \mathrm{mmol})$ was mixed with $\mathrm{CH}_{2} \mathrm{Cl}_{2}(3.8 \mathrm{~mL})$ and the mixture was added into flask dropwise at $0{ }^{\circ} \mathrm{C}$. The reaction mixture was stirred at $0{ }^{\circ} \mathrm{C}$ for $2 \mathrm{~h}$ and at room temperature for additional $24 \mathrm{~h}$. Then, aq $\mathrm{NaHCO}_{3}$ was added into flask and resulting mixture was stirred for $20 \mathrm{~min}$. Next, filtrate the mixture through a pad of celite and the solution was washed by aq $\mathrm{NaHCO}_{3}$, brine and extracted with EtOAc. Solvent was evaporated and purification by column chromatography on silica gel (hexane : EtOAc $=10: 1$ ) yielded crude 4, which was used for next step.

5

Crude $4(0.63 \mathrm{~g}, 1.53 \mathrm{mmol})$ was dissolved in benzene (4 $\mathrm{mL})$ was stirred with 3,6-diphenyl-1,2,4,5-tetrazine ( $0.40 \mathrm{~g}, 1.1$ equiv), in a sealed pressure tube at $120^{\circ} \mathrm{C}$ (oil bath) for $24 \mathrm{~h}$. After evaporation, the mixture was 
purified by column chromatography on silica gel (hexane : EtOAc $=20: 1)$ gave 5 ( $0.37 \mathrm{~g}, 0.70 \mathrm{mmol}, 19 \%$ of 3 steps) as yellow oil. ${ }^{1} \mathbf{H}$ NMR (400 MHz, $\left.\mathrm{CDCl}_{3}\right): \delta=8.31-8.28(\mathrm{~m}, 4 \mathrm{H}), 7.57-7.47(\mathrm{~m}, 6 \mathrm{H}), 3.09$ (t, $J=6.34 \mathrm{~Hz}, 4 \mathrm{H}), 1.47-1.39(\mathrm{~m}, 4 \mathrm{H}), 1.31-0.96(\mathrm{~m}, 28 \mathrm{H}), 0.87(\mathrm{t}, J=6.85 \mathrm{~Hz}, 6 \mathrm{H})$

\section{$7 / 8$}

A 2.3 M solution of $n$-BuLi in hexane $(13.0 \mathrm{~mL}, 1.5$ equiv) was added slowly to a solution of cyclopentadiene 6 (3.3 mL, 2 equiv) in THF at $-78{ }^{\circ} \mathrm{C}$ under nitrogen atmosphere. The reaction mixture was stirred at -78 ${ }^{\circ} \mathrm{C}$ for $1 \mathrm{~h}$ and then 1-bromodecane was added dropwise at $-78{ }^{\circ} \mathrm{C}$ and stirred for additional $65 \mathrm{~h}$ at room temperature. Next, the reaction was quench by ice water and then saturated $\mathrm{NH}_{4} \mathrm{Cl}$ was added to the mixture. The organic layer was washed by brine and extracted with EtOAc. After drying $\left(\mathrm{Na}_{2} \mathrm{SO}_{4}\right)$, filtration, evaporation of solvent, the residue was purified by column chromatography on silica gel (hexane only) to obtain $\mathbf{7}$ and $\mathbf{8}$ and the mixture product was directly used for next step.

\section{AZ'4b}

Under exclusion of light, to a solution of cyclopentadiene $(0.58 \mathrm{~mL}, 10$ equiv $)$ and $\mathbf{5}(0.38 \mathrm{~g}, 0.71 \mathrm{mmol})$ in $\mathrm{CH}_{2} \mathrm{Cl}_{2}(2 \mathrm{~mL})$ was added dropwise trifluoroacetic acid $\left(10.5 \mu \mathrm{L}, 0.2\right.$ equiv) at $0{ }^{\circ} \mathrm{C}$ under nitrogen atmosphere. After stirring for $2 \mathrm{~h}$, the reaction was quench by aq $\mathrm{NaHCO}_{3}$ and then organic layer was washed by brine and extracted with $\mathrm{CH}_{2} \mathrm{Cl}_{2}$. Next, the mixture was dried, filtrated, concentrated and $\mathbf{A Z} \mathbf{Z}^{\prime} \mathbf{4} \mathbf{b}(0.25 \mathrm{~g}$, $0.42 \mathrm{mmol}, 59 \%$ ) as colourless oil was prepared by purification via column chromatography on silica gel (hexane : EtOAc $=10: 1) .{ }^{1} \mathbf{H}$ NMR $\left(400 \mathrm{MHz}, \mathrm{CDCl}_{3}\right): \delta=8.00-7.88(\mathrm{~m}, 4 \mathrm{H}), 7.48-7.35(\mathrm{~m}, 6 \mathrm{H})$, 5.59-5.50 (m, 2H), 4.18-4.12 (m, 1H), 3.73-3.66 (m, 1H), 3.10-3.04 (m, 2H), 2.84-2.73 (m, 2H), 2.39-2.20 $(\mathrm{m}, 2 \mathrm{H}), 1.48-0.97(\mathrm{~m}, 32 \mathrm{H}), 0.88(\mathrm{~m}, 6 \mathrm{H})$

\section{AZ4b}

Under exclusion of light, a side-arm round flask was charged with the [4+2] cycloadduct $\mathbf{A Z} \mathbf{4} \mathbf{4 b}(0.25 \mathrm{~g}$, $0.42 \mathrm{mmol})$ dissolved in EtOAc $(4 \mathrm{~mL})$ and palladium ( $0.05 \mathrm{~g}, 1.1$ equiv) on activated carbon $10 \%$ under hydrogen gas atmosphere. After stirring $37 \mathrm{~h}$ at room temperature, the reaction mixture was filtrated over celite, concentrated and the desired compound AZ4b (0.19 g, $0.32 \mathrm{mmol}, 74 \%)$ as colourless oil was obtained by purification via column chromatography and recycle column chromatography on silica gel (hexane : EtOAc $=10: 1) .{ }^{1} \mathbf{H}$ NMR $\left(400 \mathrm{MHz}, \mathrm{CDCl}_{3}\right): \delta=7.96-7.91(\mathrm{~m}, 4 \mathrm{H}), 7.46-7.35(\mathrm{~m}, 6 \mathrm{H})$, 
$3.58-3.51(\mathrm{~m}, 2 \mathrm{H}), 3.04(\mathrm{t}, J=6.36 \mathrm{~Hz}, 2 \mathrm{H}), 2.76(\mathrm{t}, J=6.32 \mathrm{~Hz}, 2 \mathrm{H}), 1.66-1.37(\mathrm{~m}, 6 \mathrm{H}), 1.33-0.96(\mathrm{~m}$, $32 \mathrm{H}), 0.88(\mathrm{~m}, 6 \mathrm{H})$

\section{Analyses of the Azoalkane AZ5 Photolysis}

The photoreaction was carried out by subjecting AZ5 (11.1 $\mathrm{mM}$ in $\left.\mathrm{C}_{6} \mathrm{D}_{6}\right)$ to irradiation by LED lamp ( $\lambda_{\text {exc }}$ $=365 \mathrm{~nm}$ ) in a sealed NMR tube under $\mathrm{N}_{2}$ atmosphere at $298 \mathrm{~K}$. The photoproduct was directly analysis by NMR spectroscopy $\left({ }^{1} \mathrm{H},{ }^{13} \mathrm{C}\right.$, Figure S5-6) including nuclear Overhauser effect measurements (NOE, Figure S7) and FD mass spectrometry (MS, Figure S8). The results revealed the quantitative formation of the ringclosed product trans-CP5.

\section{Spectral Data}

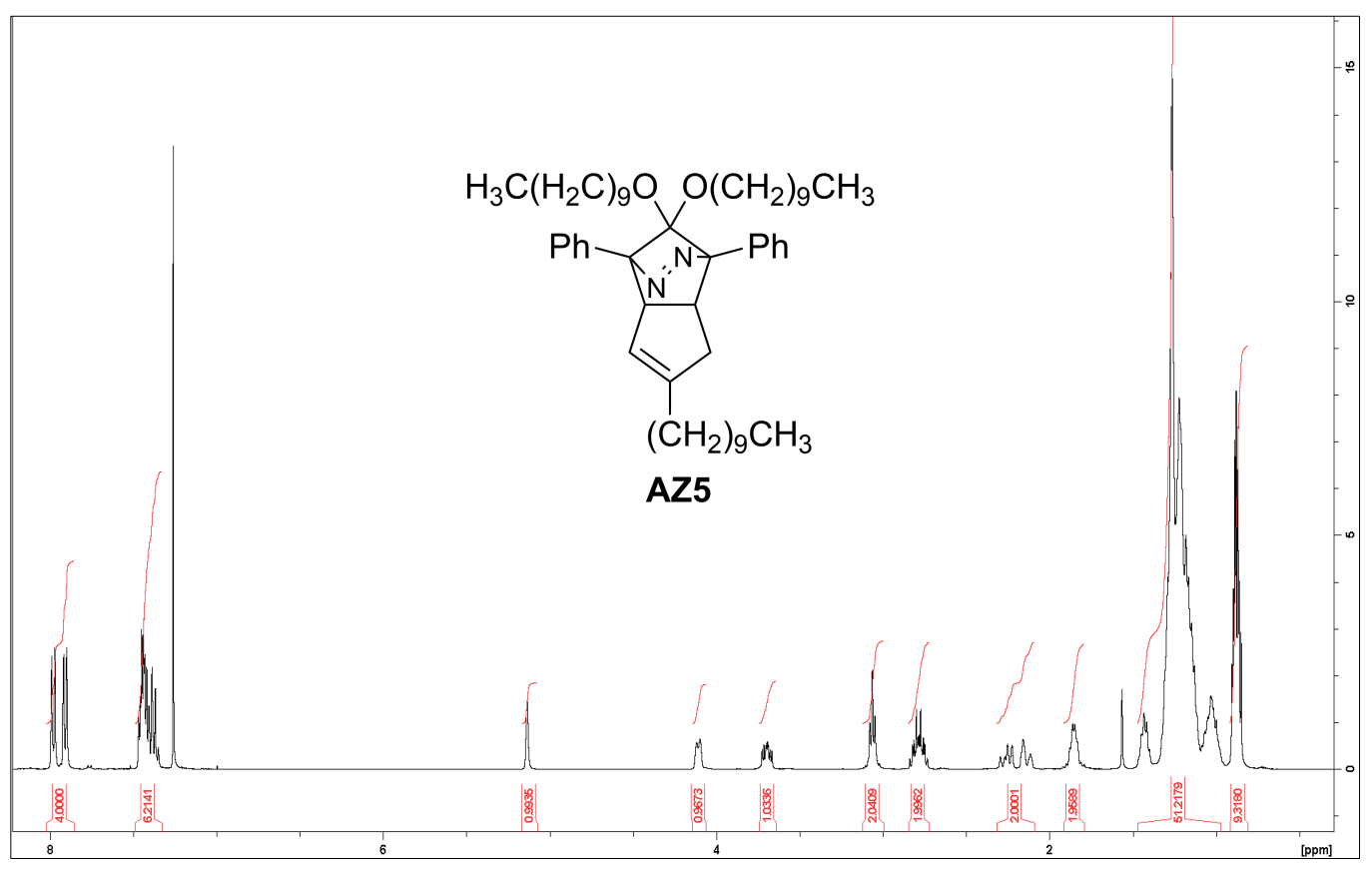

Figure S1. ${ }^{1} \mathrm{H}$ NMR spectrum of $\mathbf{A Z 5}\left(\mathrm{CDCl}_{3}, 400 \mathrm{MHz}\right)$. 


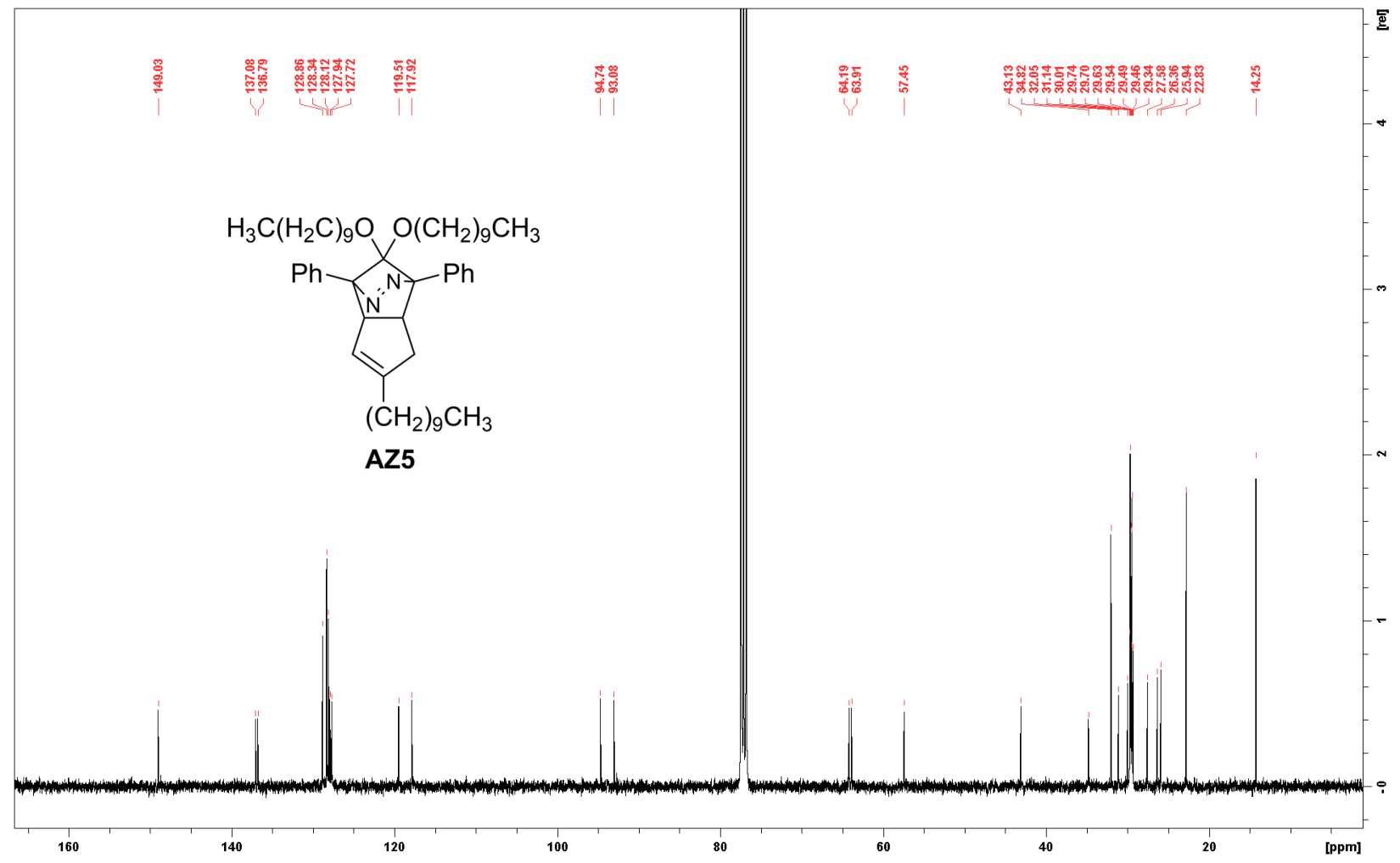

Figure S2. ${ }^{13} \mathrm{C}\left\{{ }^{1} \mathrm{H}\right\}$ NMR spectrum of $\mathbf{A Z 5}\left(\mathrm{CDCl}_{3}, 100 \mathrm{MHz}\right)$.

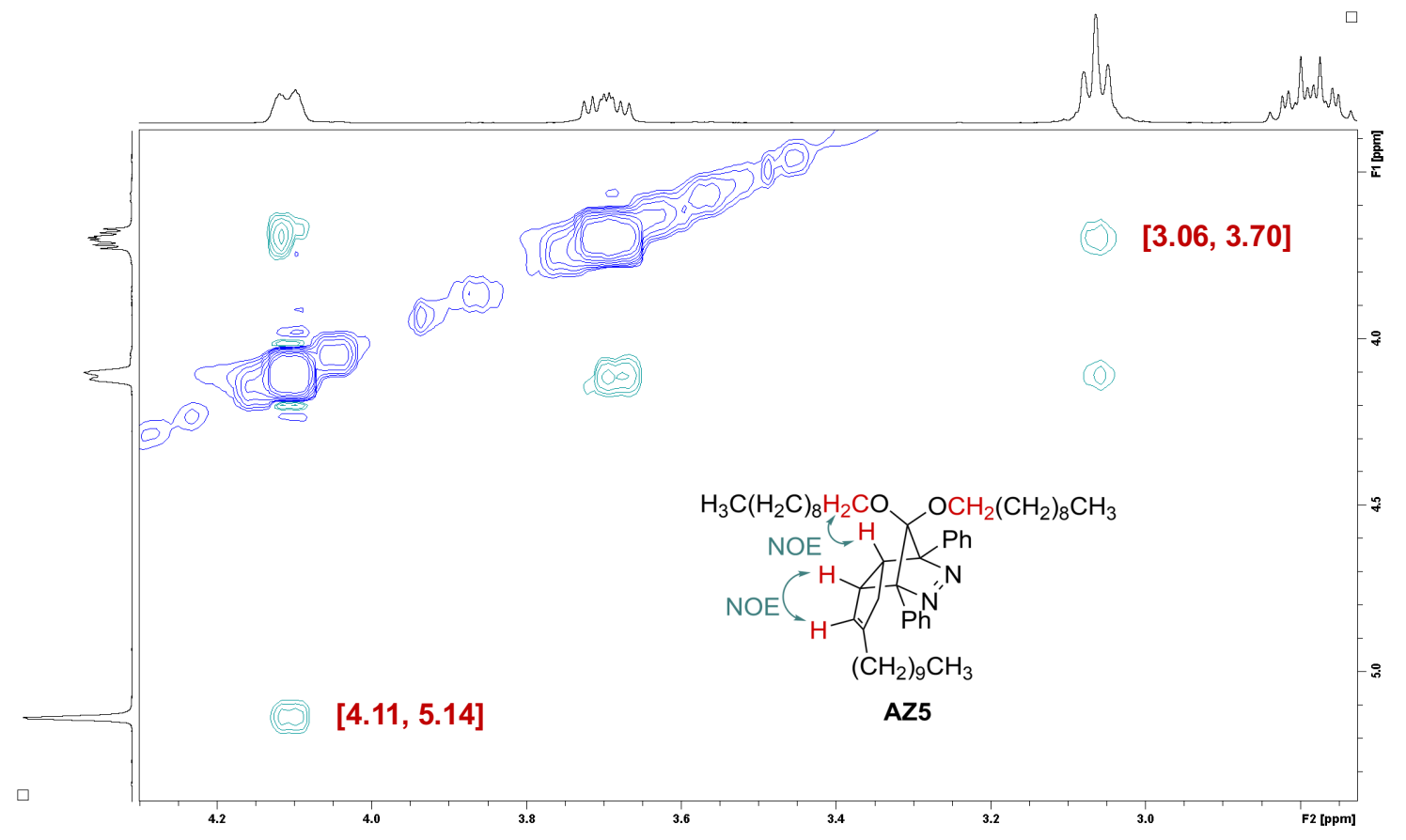

Figure S3. 2D NOESY NMR spectrum of $\mathbf{A Z 5}\left(\mathrm{CDCl}_{3}, 400 \mathrm{MHz}\right)$. 


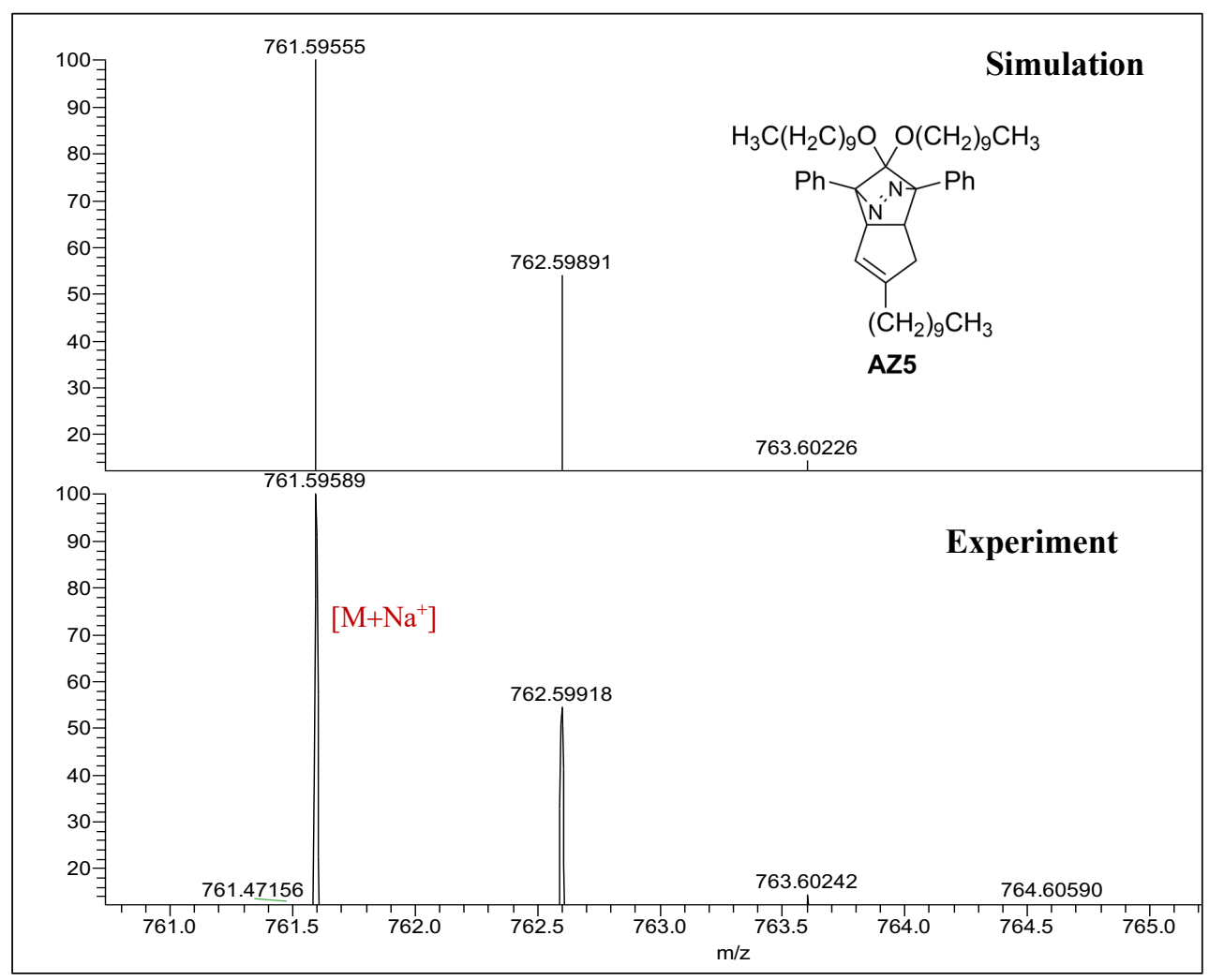

Figure S4. High resolution mass spectrum (ESI) of AZ5.

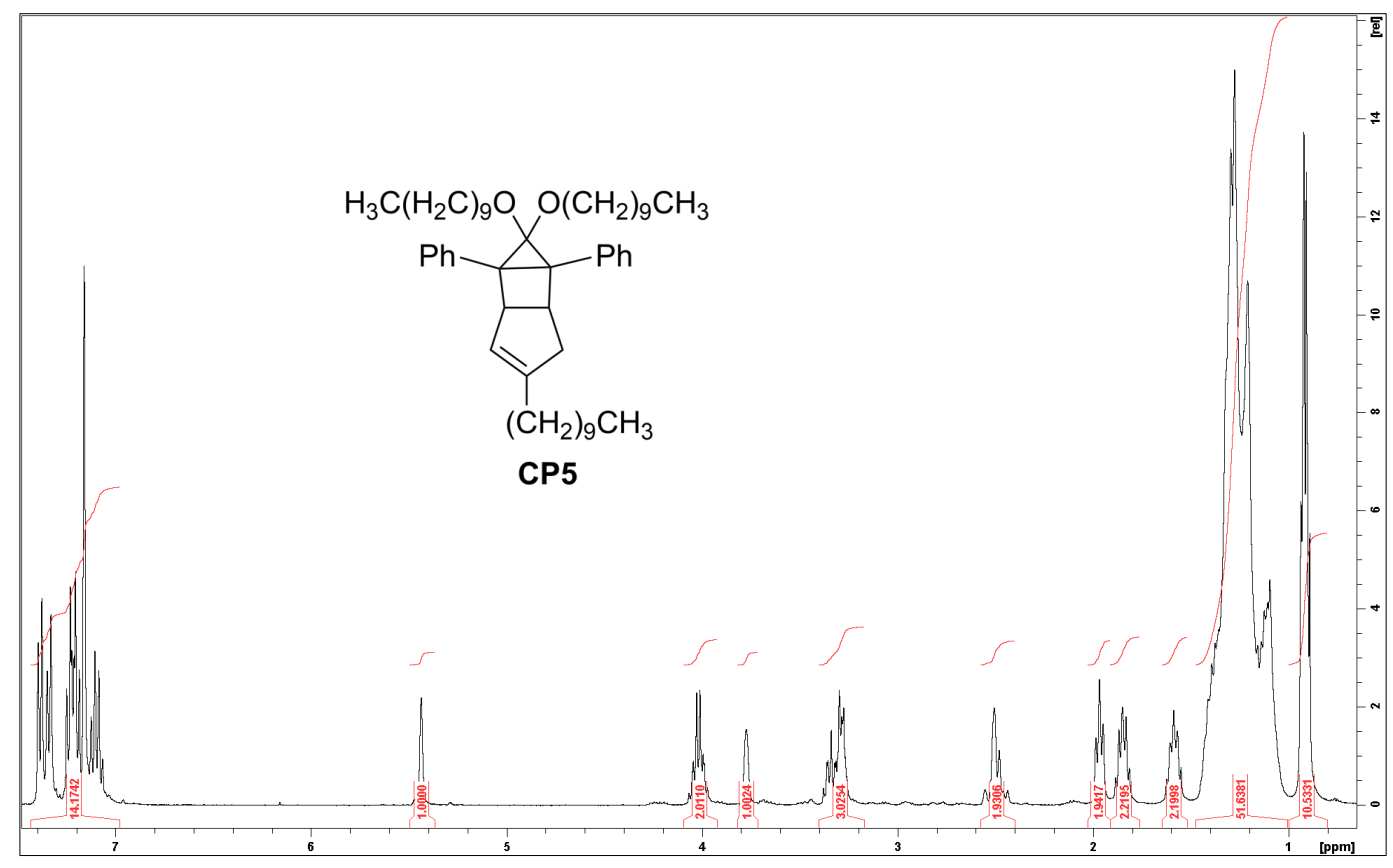


Figure S5. ${ }^{1} \mathrm{H}$ NMR spectrum of $\mathbf{C P 5}\left(\mathrm{C}_{6} \mathrm{D}_{6}, 400 \mathrm{MHz}\right)$.

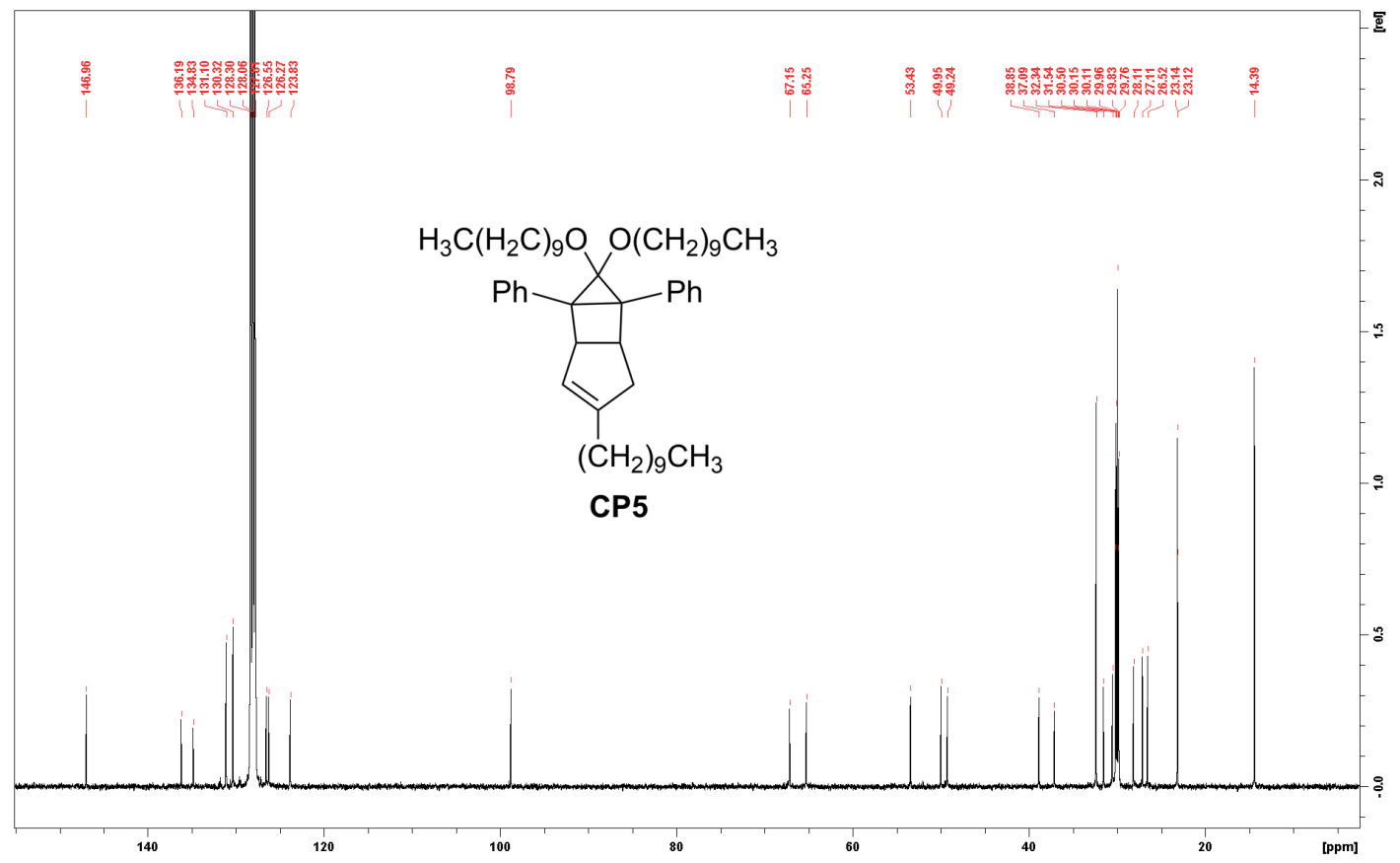

Figure S6. ${ }^{13} \mathrm{C}\left\{{ }^{1} \mathrm{H}\right\}$ NMR spectrum of $\mathbf{C P 5}\left(\mathrm{C}_{6} \mathrm{D}_{6}, 100 \mathrm{MHz}\right)$.

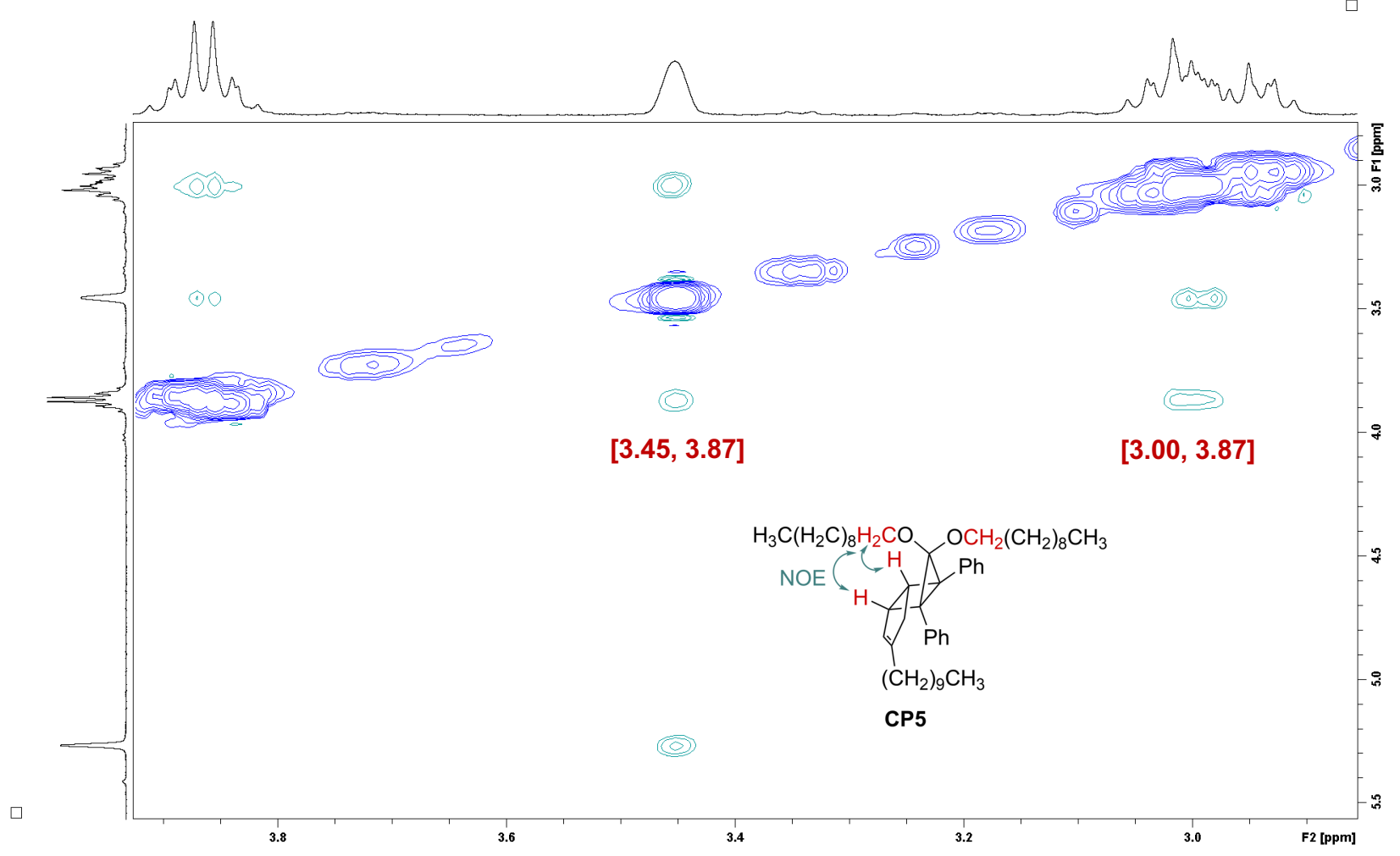

Figure S7. 2D NOESY NMR spectrum of $\mathbf{C P 5}\left(\mathrm{CDCl}_{3}, 400 \mathrm{MHz}\right)$. 


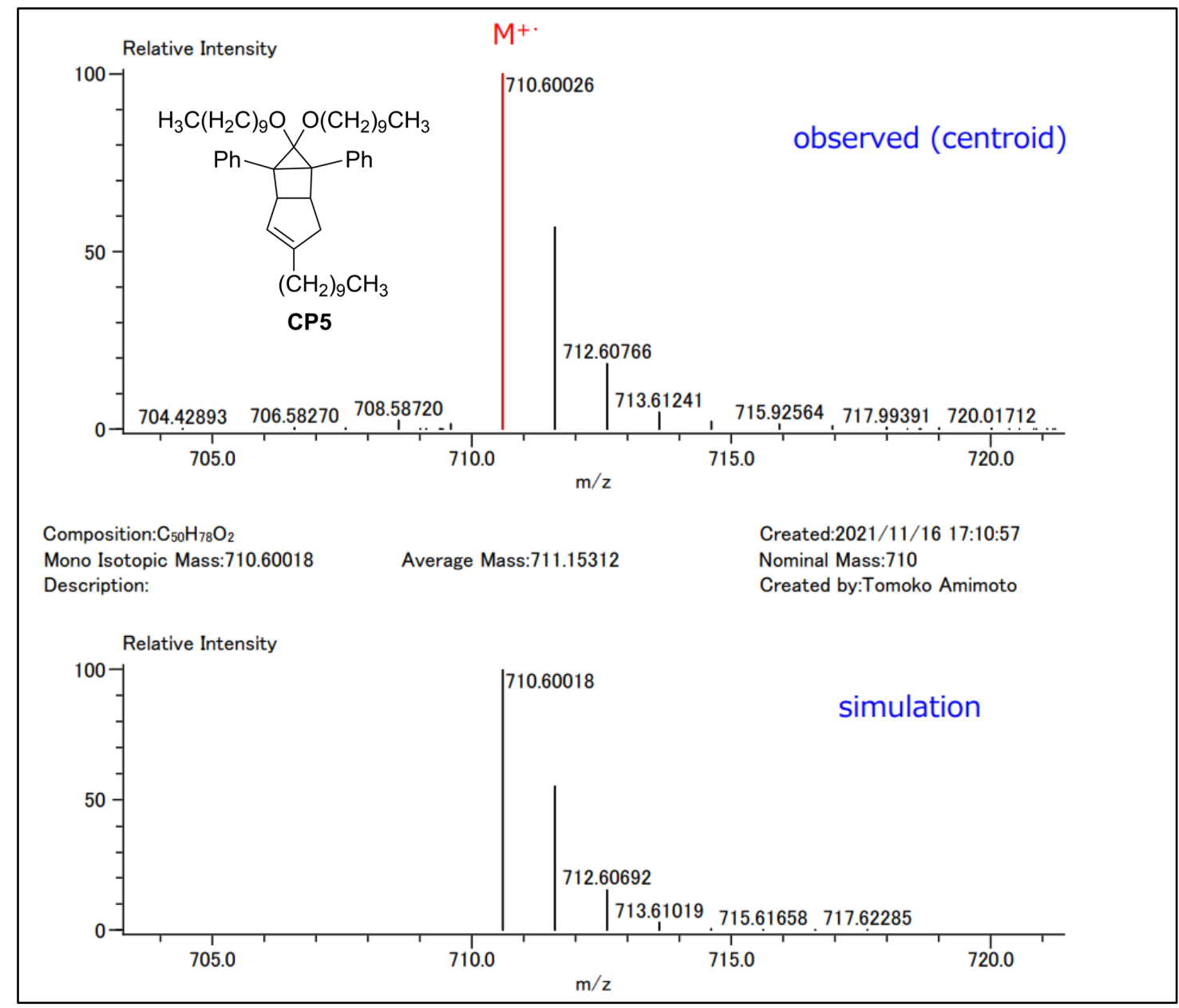

Figure S8. High resolution mass spectrum (FD) of CP5.

\section{Determination of polarity}

Table S1. Determination of polarity $\left(\pi^{*}\right)$ of binary systems containing 1-Butyl-3-methylimidazolium hexafluorophosphate ([BMIM] $\left.\left[\mathrm{PF}_{6}\right]\right)$ and glycerin triacetate (GTA)/ dimethyl sulfoxide (DMSO).

\begin{tabular}{cccc}
\hline Entry & GTA mole fraction & $\lambda_{\max } / \mathrm{nm}$ & $\pi^{*} / \mathrm{kcal} \mathrm{mol}^{-1}$ \\
\hline 1 & 0.3571 & 312.11 & 0.88 \\
2 & 0.5263 & 311.73 & 0.87 \\
3 & 0.6249 & 311.27 & 0.85 \\
4 & 0.6896 & 310.70 & 0.82 \\
5 & 0.8474 & 309.76 & 0.78 \\
\hline Entry & DMSO mole fraction & $\lambda_{\max } / \mathrm{nm}$ & $\pi^{*} / \mathrm{kcal} \mathrm{mol}^{-1}$ \\
\hline 6 & 0.1640 & 313.00 & 0.92 \\
7 & 0.3704 & 313.21 & 0.93
\end{tabular}




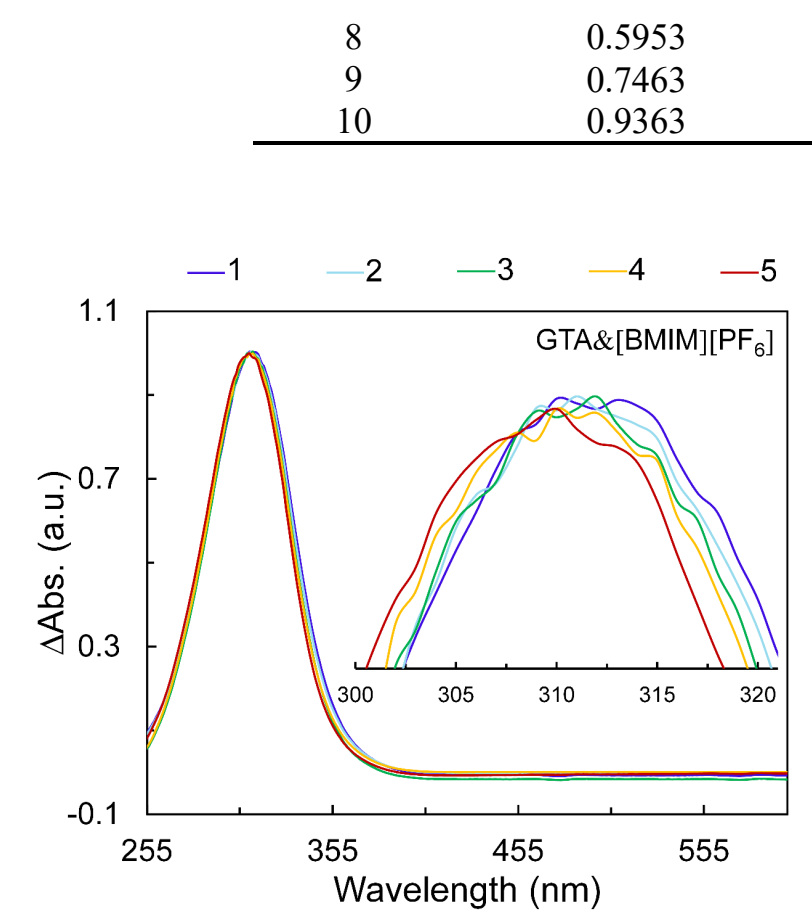

\begin{tabular}{ll}
313.68 & 0.95 \\
314.25 & 0.97 \\
314.73 & 0.99 \\
\hline
\end{tabular}

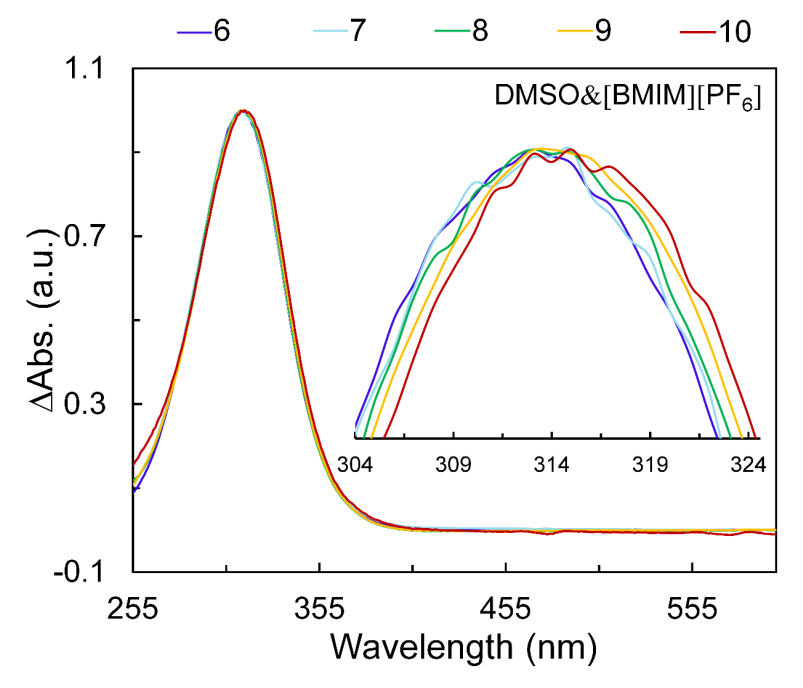

Figure S9. UV-vis absorption spectra of 4-nitroanisole in binary systems containing $[\mathrm{BMIM}]\left[\mathrm{PF}_{6}\right]$ and GTA/DMSO (inset: UV-vis absorption spectra with a step of $0.01 \mathrm{~nm}$ ) (Table S1: entries 1-10).
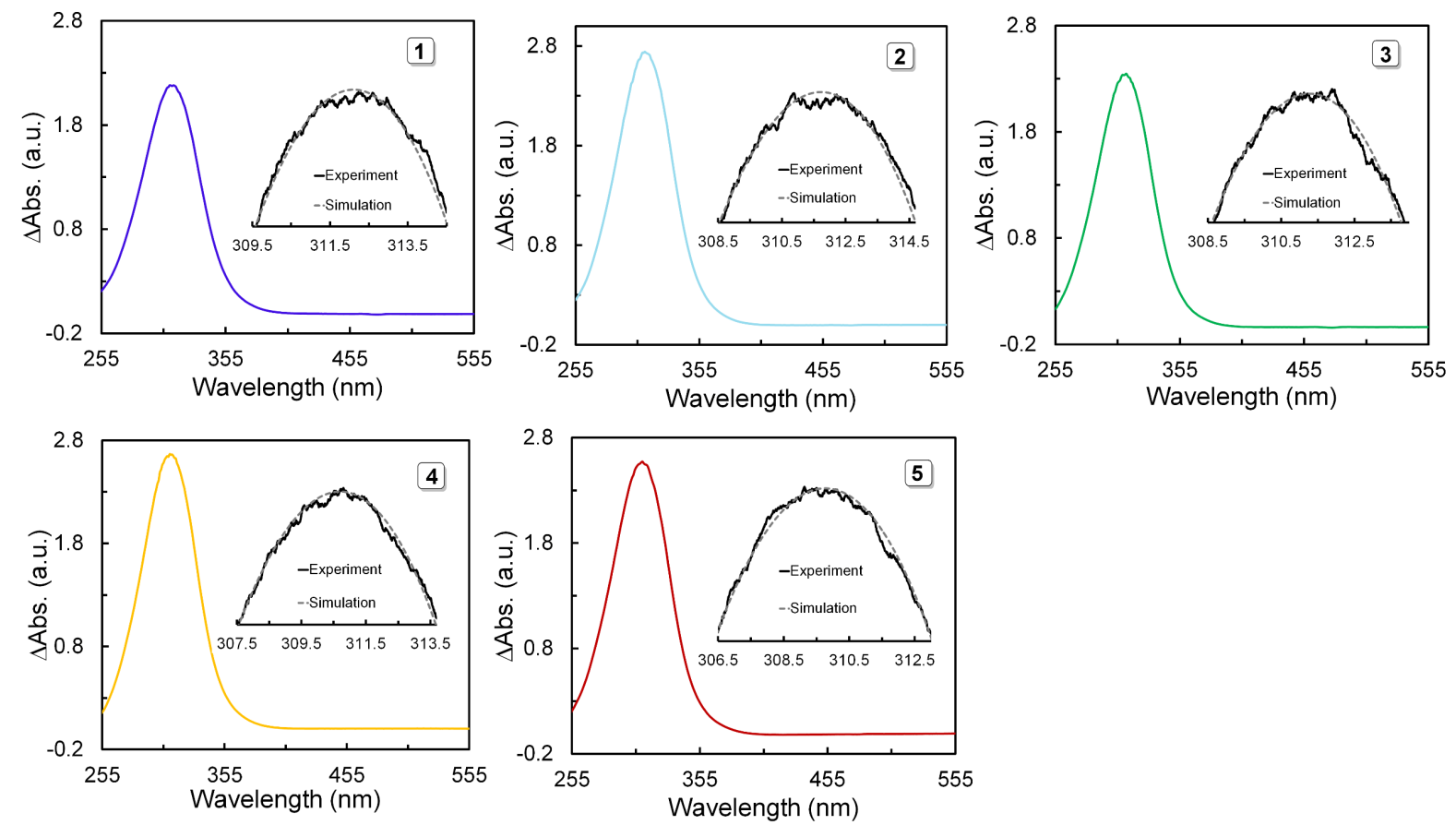

Figure S10. UV-vis absorption spectra of 4-nitroanisole in binary systems containing [BMIM][PF 6$]$ and GTA (inset: experimental and simulated UV-vis absorption spectra with a step of $0.01 \mathrm{~nm}$ ) (Table S1: entries 

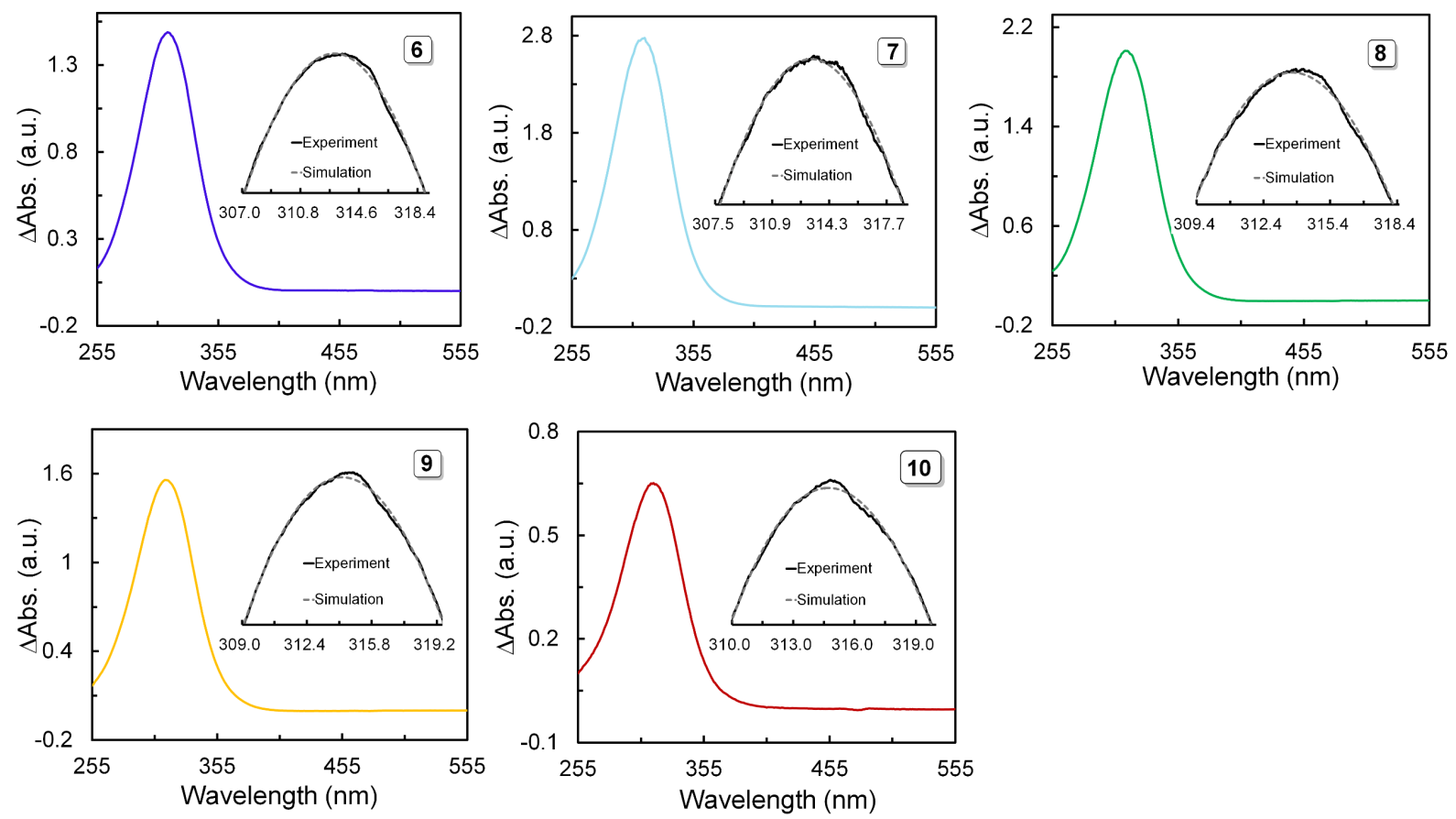

Figure S11. UV-vis absorption spectra of 4-nitroanisole in binary systems containing $[\mathrm{BMIM}]\left[\mathrm{PF}_{6}\right]$ and DMSO (inset: experimental and simulated UV-vis absorption spectra with a step of $0.01 \mathrm{~nm}$ ) (Table S1: entries 6-10).

Table S2. Equation for simulated UV-vis absorption spectra.

\begin{tabular}{|c|c|c|c|}
\hline Entry & GTA mole fraction & Equation & $R^{2}$ \\
\hline 1 & 0.3571 & $\begin{array}{c}\mathrm{y}=2.77 \mathrm{E}-08 \mathrm{x}^{6}-5.13 \mathrm{E}-05 \mathrm{x}^{5}+3.96 \mathrm{E}-02 \mathrm{x}^{4}-1.63 \mathrm{E}+01 \mathrm{x}^{3}+ \\
3.77 \mathrm{E}+03 \mathrm{x}^{2}-4.65 \mathrm{E}+05 \mathrm{x}+2.39 \mathrm{E}+07\end{array}$ & 0.9998 \\
\hline 2 & 0.5263 & $\begin{array}{c}\mathrm{y}=5.32 \mathrm{E}-08 \mathrm{x}^{6}-9.86 \mathrm{E}-05 \mathrm{x}^{5}+7.62 \mathrm{E}-02 \mathrm{x}^{4}-3.14 \mathrm{E}+01 \mathrm{x}^{3}+ \\
7.27 \mathrm{E}+03 \mathrm{x}^{2}-8.98 \mathrm{E}+05 \mathrm{x}+4.62 \mathrm{E}+07\end{array}$ & 0.9996 \\
\hline 3 & 0.6249 & $\begin{array}{c}\mathrm{y}=5.27 \mathrm{E}-08 \mathrm{x}^{6}-9.77 \mathrm{E}-05 \mathrm{x}^{5}+7.54 \mathrm{E}-02 \mathrm{x}^{4}-3.11 \mathrm{E}+01 \mathrm{x}^{3}+ \\
7.20 \mathrm{E}+03 \mathrm{x}^{2}-8.90 \mathrm{E}+05 \mathrm{x}+4.58 \mathrm{E}+07\end{array}$ & 0.9995 \\
\hline 4 & 0.6896 & $\begin{array}{c}y=5.11 E-08 x^{6}-9.47 E-05 x^{5}+7.32 E-02 x^{4}-3.01 E+01 x^{3}+ \\
6.98 E+03 x^{2}-8.63 E+05 x+4.44 E+07\end{array}$ & 0.9992 \\
\hline 5 & 0.8474 & $\begin{array}{c}\mathrm{y}=6.09 \mathrm{E}-08 \mathrm{x}^{6}-1.13 \mathrm{E}-04 \mathrm{x}^{5}+8.73 \mathrm{E}-02 \mathrm{x}^{4}-3.60 \mathrm{E}+01 \mathrm{x}^{3}+ \\
8.34 \mathrm{E}+03 \mathrm{x}^{2}-1.03 \mathrm{E}+06 \mathrm{x}+5.31 \mathrm{E}+07\end{array}$ & 0.9991 \\
\hline Entry & DMSO mole fraction & Equation & $R^{2}$ \\
\hline 6 & 0.1640 & $\begin{array}{c}\mathrm{y}=-1.18 \mathrm{E}-08 \mathrm{x}^{6}+2.21 \mathrm{E}-05 \mathrm{x}^{5}-1.73 \mathrm{E}-02 \mathrm{x}^{4}+7.20 \mathrm{E}+00 \mathrm{x}^{3} \\
-1.69 \mathrm{E}+03 \mathrm{x}^{2}+2.11 \mathrm{E}+05 \mathrm{x}-1.10 \mathrm{E}+07\end{array}$ & 0.9996 \\
\hline 7 & 0.3704 & $\begin{array}{c}\mathrm{y}=-3.91 \mathrm{E}-08 \mathrm{x}^{6}+7.35 \mathrm{E}-05 \mathrm{x}^{5}-5.75 \mathrm{E}-02 \mathrm{x}^{4}+2.40 \mathrm{E}+01 \mathrm{x}^{3} \\
-5.62 \mathrm{E}+03 \mathrm{x}^{2}+7.04 \mathrm{E}+05 \mathrm{x}-3.67 \mathrm{E}+07\end{array}$ & 0.9994 \\
\hline 8 & 0.5953 & $\begin{array}{c}\mathrm{y}=-1.74 \mathrm{E}-08 \mathrm{x}^{6}+3.26 \mathrm{E}-05 \mathrm{x}^{5}-2.55 \mathrm{E}-02 \mathrm{x}^{4}+1.06 \mathrm{E}+01 \mathrm{x}^{3} \\
-2.48 \mathrm{E}+03 \mathrm{x}^{2}+3.10 \mathrm{E}+05 \mathrm{x}-1.61 \mathrm{E}+07\end{array}$ & 0.9998 \\
\hline 9 & 0.7463 & $\begin{array}{c}\mathrm{y}=-1.27 \mathrm{E}-08 \mathrm{x}^{6}+2.39 \mathrm{E}-05 \mathrm{x}^{5}-1.87 \mathrm{E}-02 \mathrm{x}^{4}+7.80 \mathrm{E}+00 \mathrm{x}^{3} \\
-1.83 \mathrm{E}+03 \mathrm{x}^{2}+2.29 \mathrm{E}+05 \mathrm{x}-1.19 \mathrm{E}+07\end{array}$ & 0.9998 \\
\hline
\end{tabular}




\section{Regression statistics}

Table S3. Regression statistics for S-DR4a, S-DR4b and S-DR5 in different pure organic solvents.

\begin{tabular}{lllllllll}
\hline & & Standard & & & & Upper & Lower & Upper \\
S-DR4a & Coefficients & Error & t Stat & P-value & Lower $95 \%$ & $95 \%$ & $95.0 \%$ & $95.0 \%$ \\
\hline Intercept & -15.7681 & 0.0501 & -314.6635 & $1.1541 \mathrm{E}-49$ & -15.8709 & -15.6653 & -15.8709 & -15.6653 \\
Polarity variable & 1.4812 & 0.0825 & 17.9532 & $1.5430 \mathrm{E}-16$ & 1.3119 & 1.6504 & 1.3119 & 1.6504 \\
Viscosity variable & 0.0248 & 0.0020 & 12.5940 & $8.1499 \mathrm{E}-13$ & 0.0207 & 0.0288 & 0.0207 & 0.0288 \\
\hline & & Standard & & & & Upper & Lower & Upper \\
S-DR4b & Coefficients & Error & t Stat & P-value & Lower $95 \%$ & $95 \%$ & $95.0 \%$ & $95.0 \%$ \\
\hline Intercept & -13.4965 & 0.0637 & -211.9693 & $4.9303 \mathrm{E}-45$ & -13.6271 & -13.3658 & -13.6271 & -13.3658 \\
Polarity variable & 1.2265 & 0.1048 & 11.7002 & $4.4178 \mathrm{E}-12$ & 1.0114 & 1.4416 & 1.0114 & 1.4416 \\
Viscosity variable & 0.0252 & 0.0025 & 10.0762 & $1.2062 \mathrm{E}-10$ & 0.0201 & 0.0303 & 0.0201 & 0.0303 \\
\hline & & Standard & & & & Upper & Lower & \\
S-DR5 & Coefficients & Error & $\mathrm{t}$ Stat & P-value & Lower $95 \%$ & $95 \%$ & $95.0 \%$ & S-DR4b \\
\hline Intercept & -13.5919 & 0.0747 & -181.9138 & $3.3841 \mathrm{E}-39$ & -13.7461 & -13.4377 & -13.7461 & -13.4377 \\
Polarity variable & 1.4518 & 0.1418 & 10.2364 & $3.1086 \mathrm{E}-10$ & 1.1591 & 1.7445 & 1.1591 & 1.7445 \\
Viscosity variable & 0.0384 & 0.0028 & 13.8840 & $5.7730 \mathrm{E}-13$ & 0.0327 & 0.0441 & 0.0327 & 0.0441 \\
\hline
\end{tabular}

Table S4. Regression statistics for S-DR4a and S-DR4b in different solvents and solvent mixtures.

\begin{tabular}{|c|c|c|c|c|c|c|c|c|}
\hline & & Standard & & & & Upper & Lower & Upper \\
\hline S-DR4a & Coefficients & Error & t Stat & P-value & Lower $95 \%$ & $95 \%$ & $95.0 \%$ & $95.0 \%$ \\
\hline Intercept & -15.6907 & 0.0887 & -176.8184 & $8.8605 \mathrm{E}-73$ & -15.8688 & -15.5125 & -15.8688 & -15.5125 \\
\hline Polarity variable & 1.6273 & 0.1284 & 12.6756 & $2.1979 \mathrm{E}-17$ & 1.3695 & 1.8850 & 1.3695 & 1.8850 \\
\hline \multirow[t]{2}{*}{ Viscosity variable } & 0.0101 & 0.0011 & 9.4054 & $9.9952 \mathrm{E}-13$ & 0.0079 & 0.0123 & 0.0079 & 0.0123 \\
\hline & & Standard & & & & Upper & Lower & Upper \\
\hline S-DR4b & Coefficients & Error & t Stat & P-value & Lower $95 \%$ & $95 \%$ & $95.0 \%$ & $95.0 \%$ \\
\hline Intercept & -13.4097 & 0.0958 & -139.9987 & $1.2838 \mathrm{E}-67$ & -13.6020 & -13.2174 & -13.6020 & -13.2174 \\
\hline Polarity variable & 1.3753 & 0.1386 & 9.9250 & $1.6665 \mathrm{E}-13$ & 1.0971 & 1.6535 & 1.0971 & 1.6535 \\
\hline Viscosity variable & 0.0089 & 0.0012 & 7.6972 & $4.3327 \mathrm{E}-10$ & 0.0066 & 0.0113 & 0.0066 & 0.0113 \\
\hline
\end{tabular}

\section{Transient absorption spectra}



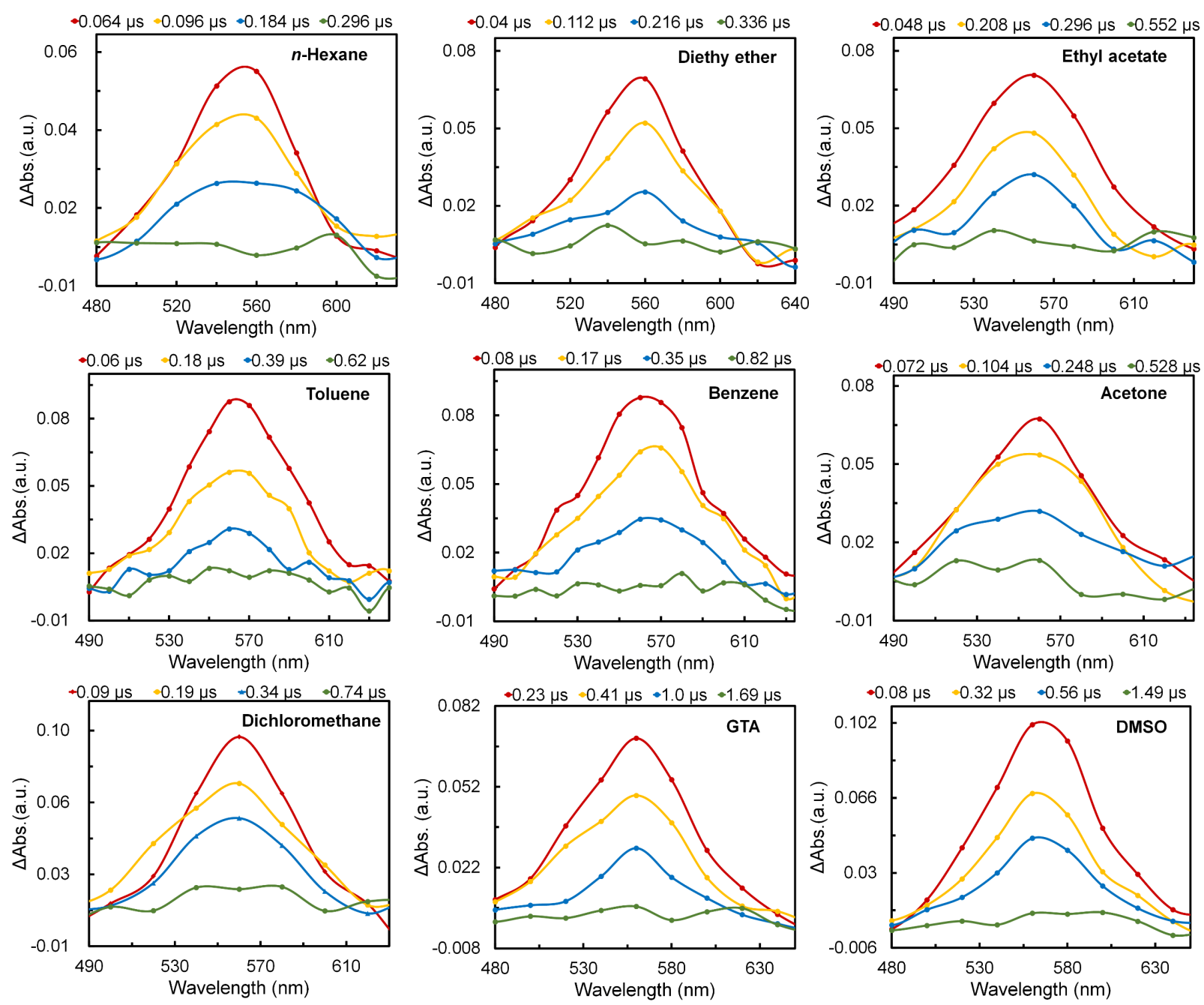

Figure S12. Sub-microsecond transient absorption spectra during the laser flash photolysis of AZ4a $\left(\lambda_{\mathrm{emi}}=\right.$ $355 \mathrm{~nm}$ ) at $293 \mathrm{~K}$ under nitrogen in $n$-hexane, diethyl ether, ethyl acetate, toluene, benzene, acetone, dichloromethane, glycerin triacetin (GTA) and dimethyl sulfoxide (DMSO). 

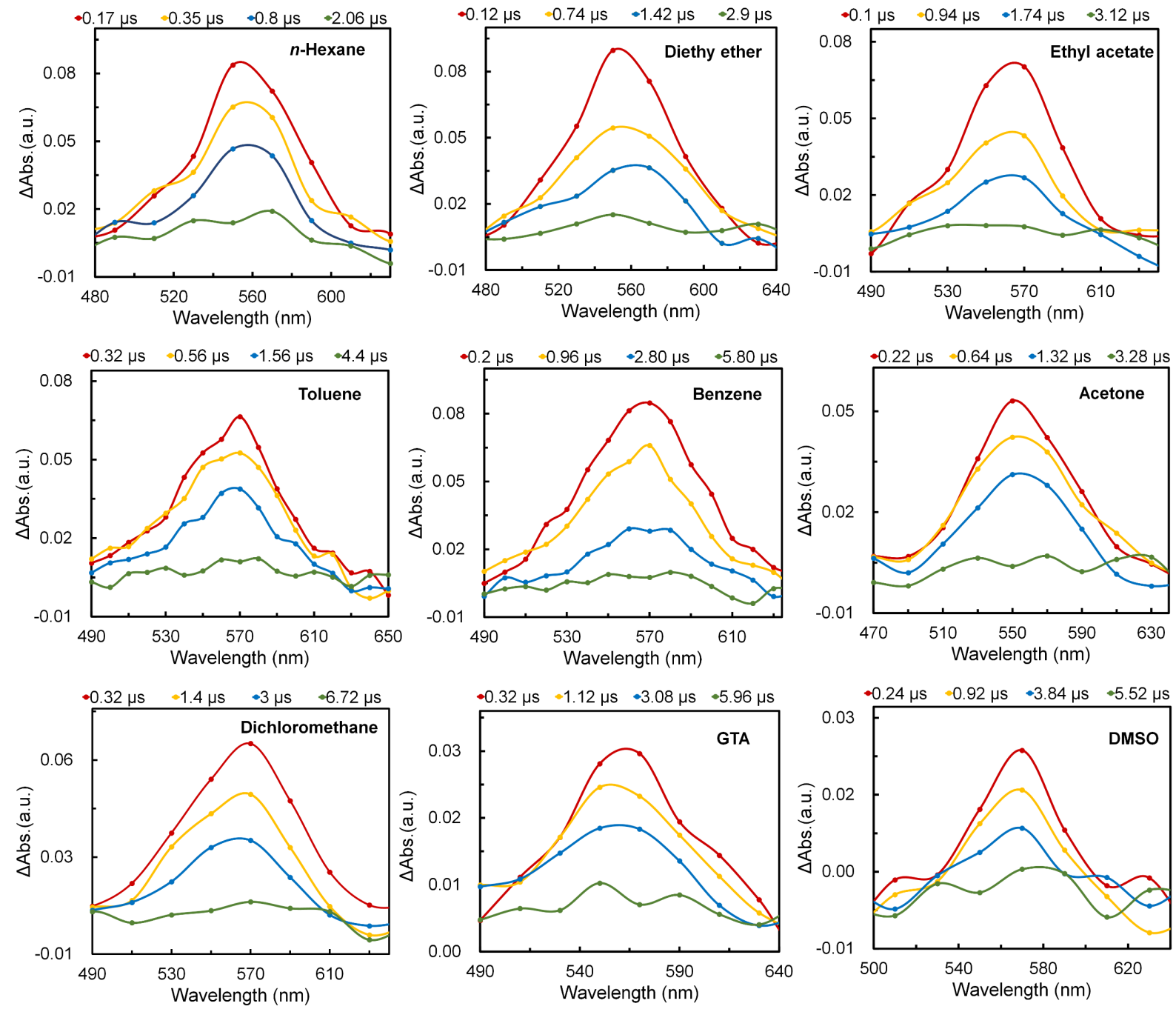

Figure S13. Sub-microsecond transient absorption spectra during the laser flash photolysis of $\mathbf{A Z 4 b}\left(\lambda_{\mathrm{emi}}=\right.$ $355 \mathrm{~nm}$ ) at $293 \mathrm{~K}$ under nitrogen in $n$-hexane, diethyl ether, ethyl acetate, toluene, benzene, acetone, dichloromethane, glycerin triacetin (GTA) and dimethyl sulfoxide (DMSO). 

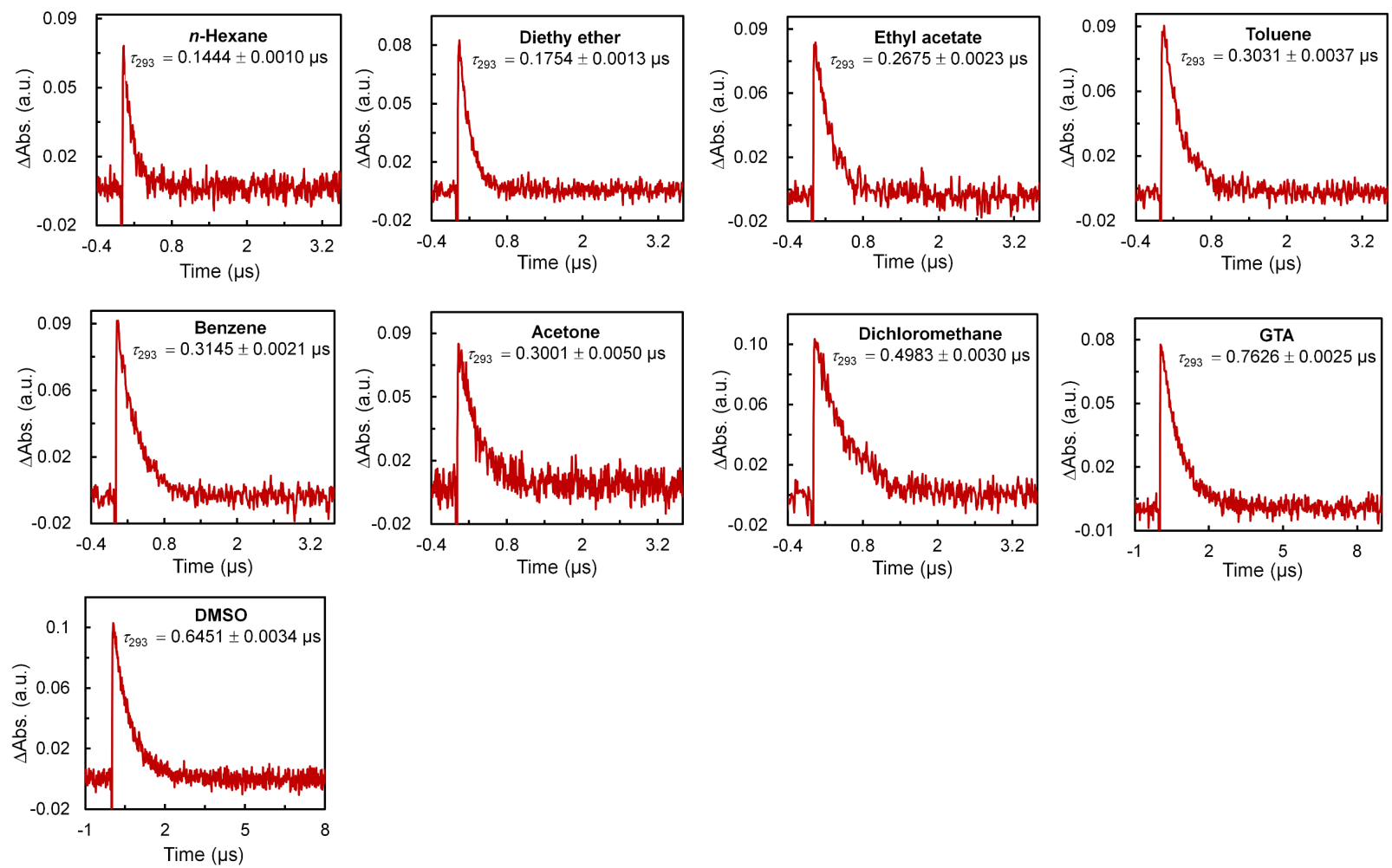

Figure S14. Time profiles at $560 \mathrm{~nm}$ of S-DR4a at $293 \mathrm{~K}$ under nitrogen in $n$-hexane, diethyl ether, ethyl acetate, toluene, benzene, acetone, dichloromethane, glycerin triacetin (GTA) and dimethyl sulfoxide (DMSO). Lifetime are calculated from single-exponential decay model fitting. 

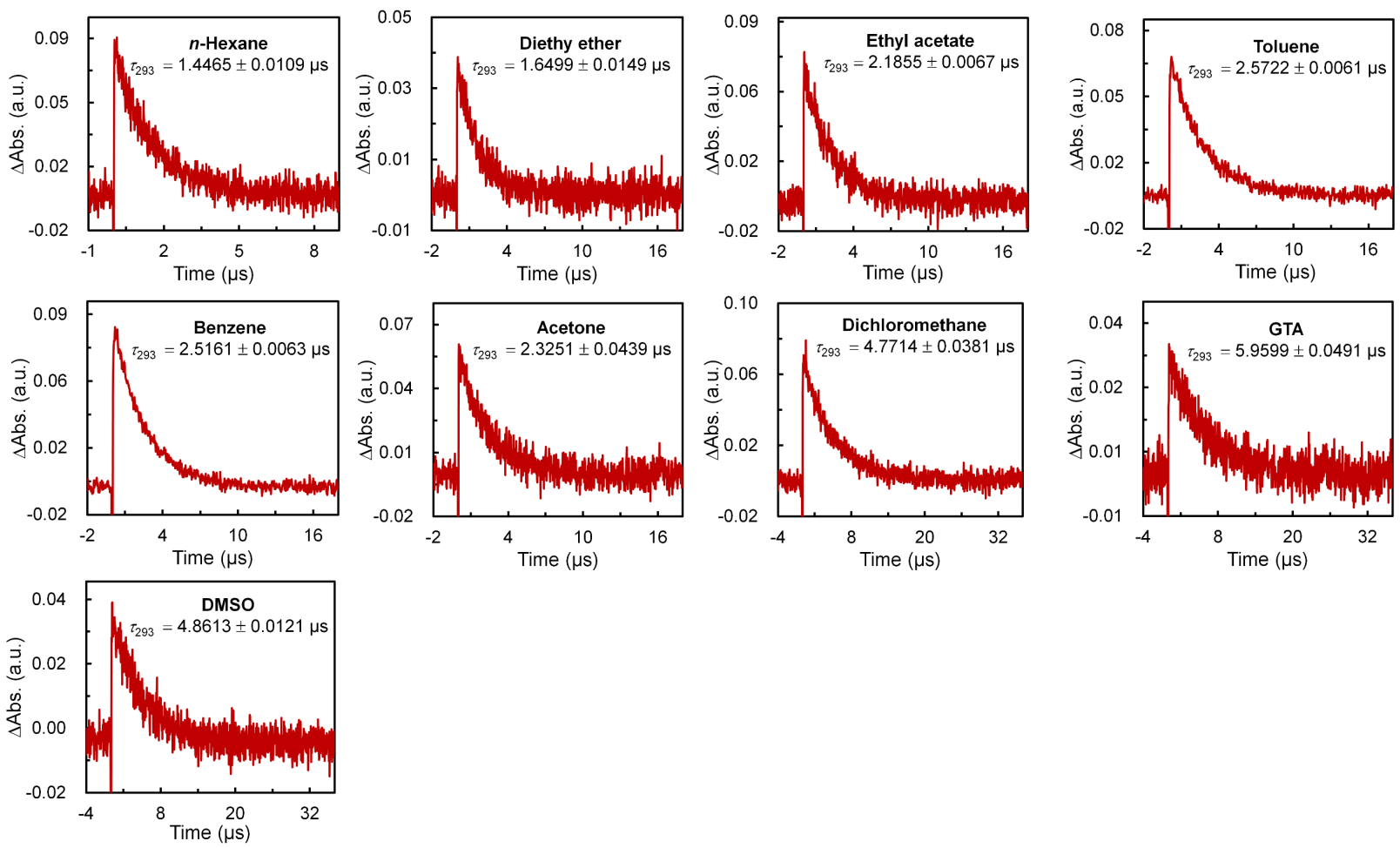

Figure S15. Time profiles at $570 \mathrm{~nm}$ of S-DR4b at $293 \mathrm{~K}$ under nitrogen in $n$-hexane, diethyl ether, ethyl acetate, toluene, benzene, acetone, dichloromethane, glycerin triacetin (GTA) and dimethyl sulfoxide (DMSO). Lifetime are calculated from single-exponential decay model fitting. 

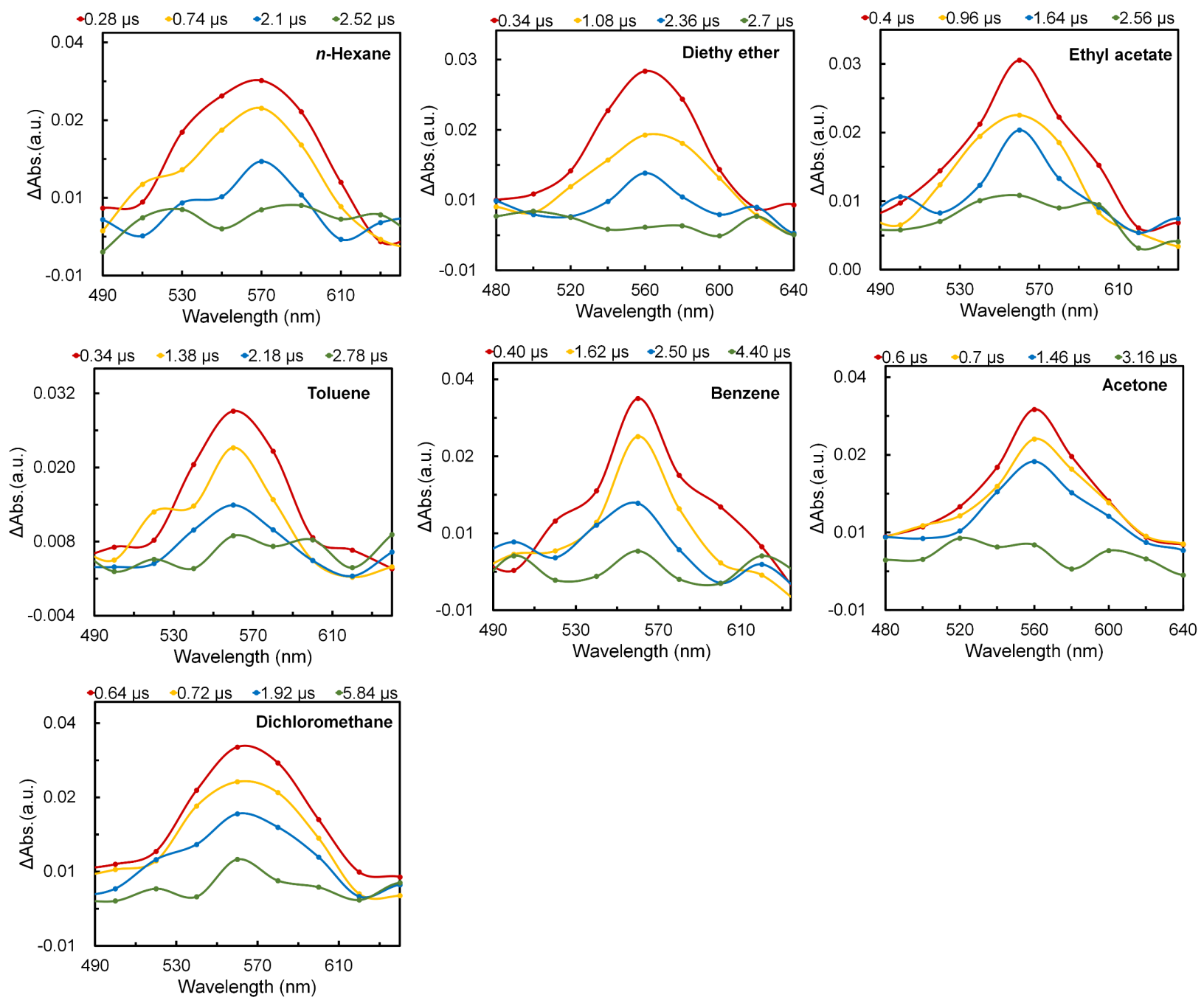

Figure S16. Sub-microsecond transient absorption spectra during the laser flash photolysis of $\mathbf{A Z 5}\left(\lambda_{\text {emi }}=355\right.$ $\mathrm{nm})$ at $293 \mathrm{~K}$ under nitrogen in $n$-hexane, diethyl ether, ethyl acetate, toluene, benzene, acetone and dichloromethane. 

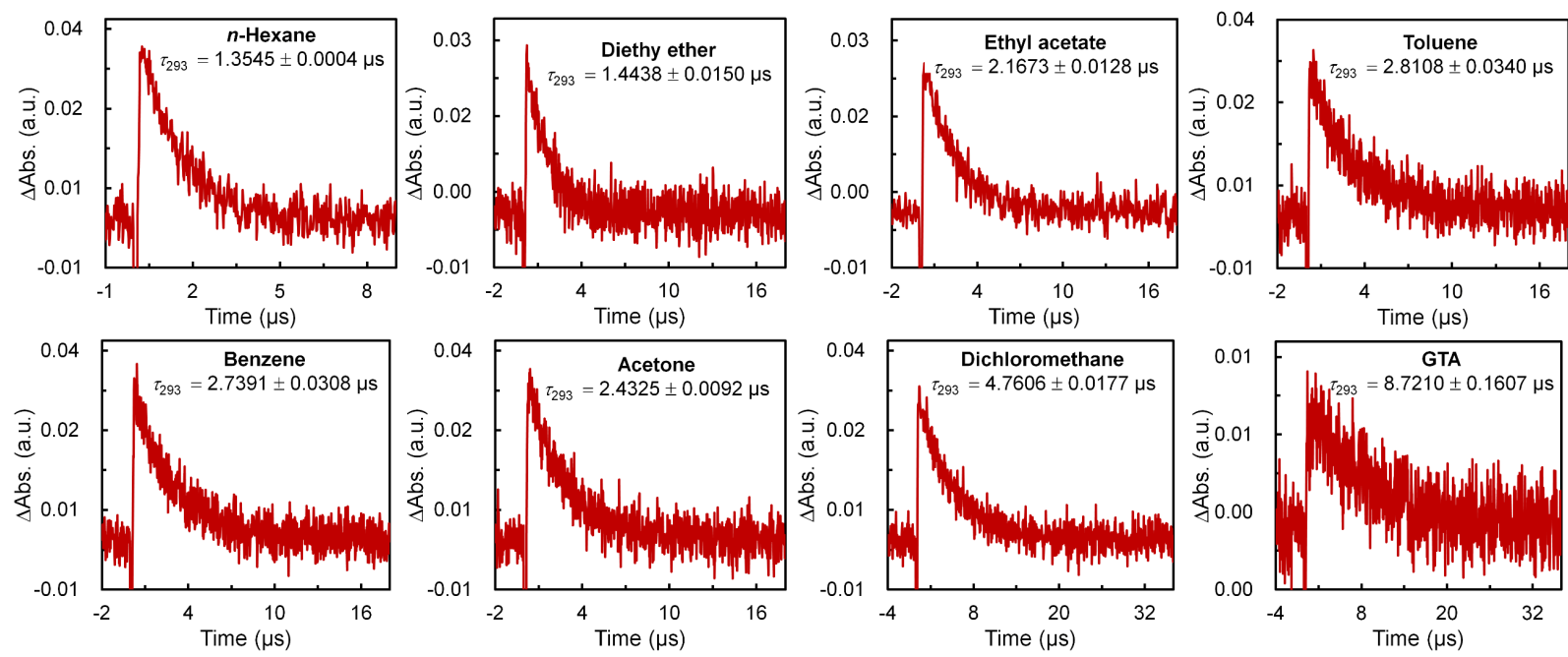

Figure S17. Time profiles at $560 \mathrm{~nm}$ of S-DR5 at $293 \mathrm{~K}$ under nitrogen in $n$-hexane, diethyl ether, ethyl acetate, toluene, benzene, acetone, dichloromethane and glycerin triacetin (GTA). Lifetime are calculated from singleexponential decay model fitting.
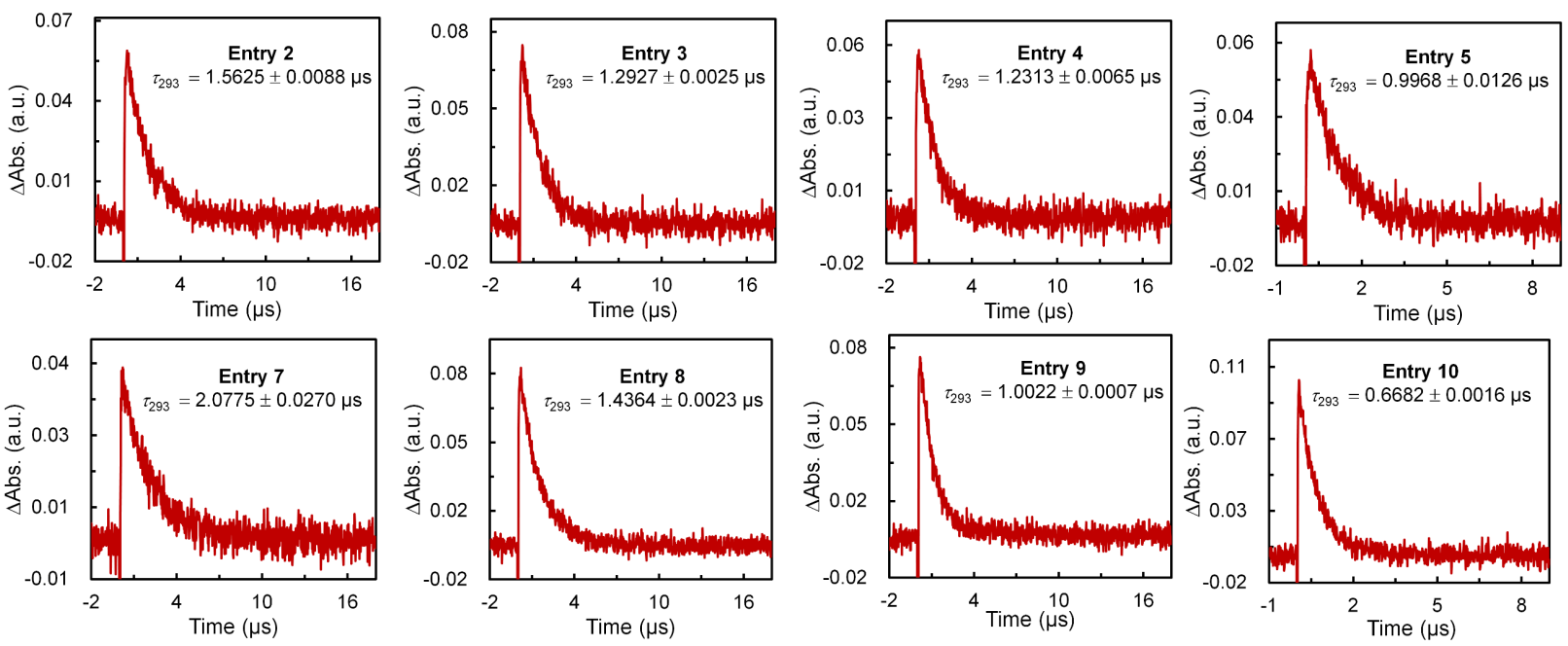

Figure S18. Time profiles at $560 \mathrm{~nm}$ of S-DR4a at $293 \mathrm{~K}$ under nitrogen in binary systems containing $[\mathrm{BMIM}]\left[\mathrm{PF}_{6}\right]$ and GTA/DMSO (Table S1: entries 2-5 and 7-10). Lifetime are calculated from singleexponential decay model fitting. 

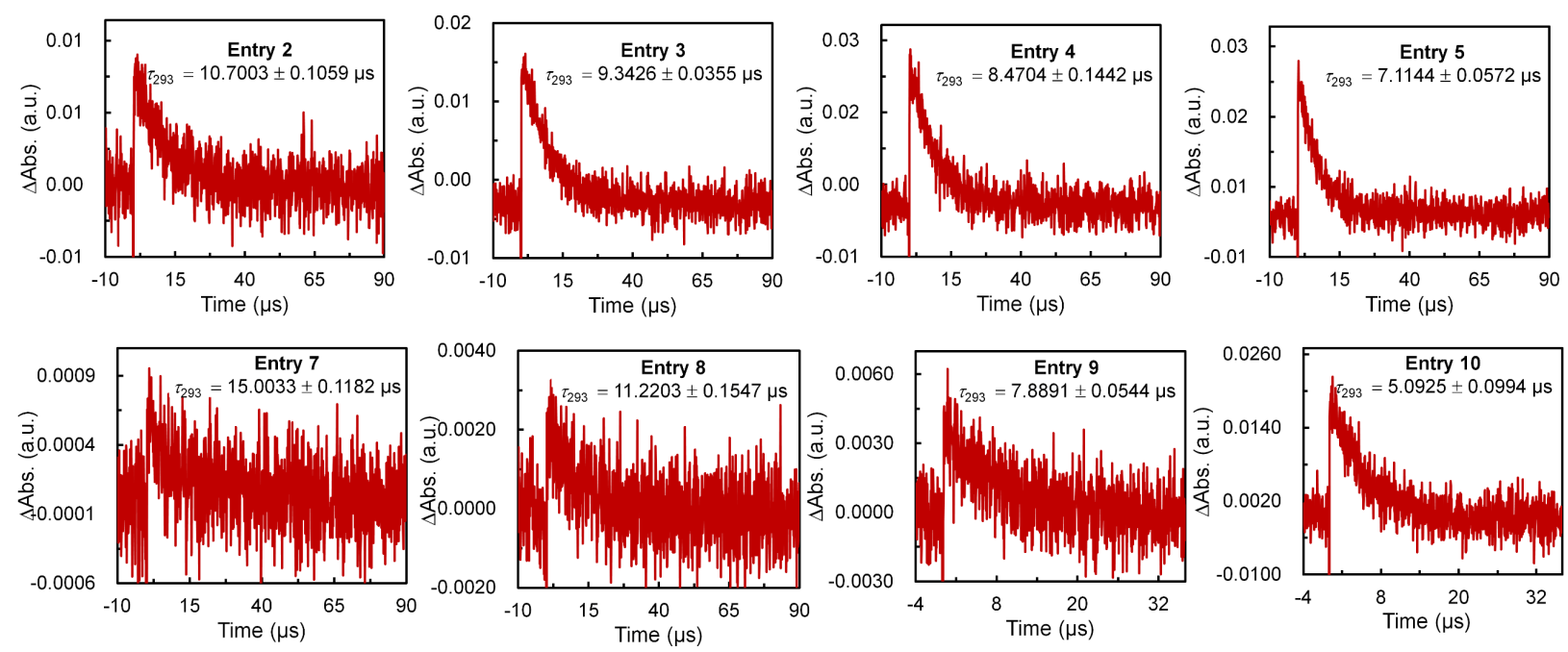

Figure S19. Time profiles at $570 \mathrm{~nm}$ of S-DR4b at $293 \mathrm{~K}$ under nitrogen in binary systems containing $[\mathrm{BMIM}]\left[\mathrm{PF}_{6}\right]$ and $\mathrm{GTA} / \mathrm{DMSO}$ (Table S1: entries $2-5$ and $7-10$ ). Lifetime are calculated from singleexponential decay model fitting. 